\title{
Aktueller Stellenwert von Erlotinib und Gefitinib in der palliativen Therapie des NSCLC - welche Bedeutung hat der EGF-R-Mutationsstatus?
}

\author{
Current Status of Erlotinib and Gefitinib in Palliative Therapy for NSCLC - \\ Does the EGF-R Mutation State have any Significance?
}

Autoren

Institute
W. M. Brückl ${ }^{1}$, G. H. Wiest ${ }^{2}$, J. H. Ficker ${ }^{1}$

Medizinische Klinik 3/Lungentumorzentrum, Klinikum Nürnberg (Chefarzt: Prof. Dr. med. J. H. Ficker)

${ }^{2}$ Lungenabteilung, Thoraxzentrum Hamburg, Asklepios Klinik Harburg (Chefarzt: PD Dr. med. G. H. Wiest) eingereicht 30.3 .2010 akzeptiert nach Revision 20. 5. 2010

\section{Bibliografie}

Dol http://dx.doi.org/ 10.1055/s-0030-1255532

Online-Publikation: 24. 6. 2010

Pneumologie 2010; 64:

727-735 @ Georg Thieme

Verlag KG Stuttgart · New York ISSN 0934-8387

\section{Korrespondenzadresse \\ PD Dr. med.}

\section{Wolfgang M. Brückl}

Leiter der pneumologischen Onkologie

Medizinische Klinik 3/ Lungentumorzentrum Prof.-Ernst-Nathan-Str. 1 90419 Nürnberg wolfgang.brueckl@Klinikumnuernberg.de

\section{Zusammenfassung \\ $\nabla$}

Die Prognose des metastasierten nichtkleinzelligen Lungenkarzinoms (NSCLC) ist trotz intensiver Forschung und ungezählter Chemotherapiestudien nach wie vor sehr schlecht, sodass zunehmend auch Therapieformen mit innovativen Wirkmechanismen eingesetzt werden. Mit Erlotinib und Gefitinib sind zwei Tyrosinkinase-Inhibitoren (TKI) zugelassen, die gezielt gegen den EGFRezeptor (EGF-R) gerichtet sind. Aktivierende Mutationen in der Tyrosinkinase-Domäne führen zu einer besseren Wirksamkeit der Substanzen. Welche klinische Bedeutung hat daher die EGFR-Mutationsbestimmung im Tumor? Wie ist der Stellenwert der TKI in der Erst-, Zweit- und Drittlinientherapie? Welche Therapieoptionen gibt es nach Versagen der TKI-Erstlinientherapie? Diese und andere in der Praxis relevante Fragen im $\mathrm{Zu}$ sammenhang mit TKI werden in diesem Übersichtsartikel diskutiert.

\section{Glossar häufig verwendeter Abkürzungen} $\nabla$

NSCLC, nichtkleinzelliges Lungenkarzinom; EGF-R, Rezeptor des epidermalen Wachstumsfaktors (epidermal growth factor receptor); VEGF, vaskulärer epithelialer Wachstumsfaktor (vascular endothelial growth factor); TKI, Tyrosinkinase Inhibitor; PS, Allgemeinzustand (performance status); PFS, progressionsfreies Überleben (progression free survival); OS, Gesamtüberleben (overall survival); WT, Wildtyp (keine Mutation) (wild type)

\section{Einleitung \\ $\nabla$}

Das Lungenkarzinom stellt die häufigste Krebstodesursache in der westlichen Welt dar. Über 40000 Menschen sterben allein in Deutschland jährlich an den Folgen der Erkrankung [1]. Lungenkarzinome werden in kleinzellige (SCLC) und

\section{Abstract \\ $\nabla$}

In spite of intensive research and a huge amount of chemotherapy trials, the prognosis of metastastic non-small cell lung cancer (NSCLC) is still poor. Erlotinib and Gefitinb are tyrosine kinase inhibitors (TKIs) which act against the EGF receptor (EGF-R). Activation of mutations in the tyrosine kinase domain leads to an increase in effectiveness. What is the clinical impact of EGF-R mutation screening? What value do TKIs in $1^{\text {st }}, 2^{\text {nd }}$ and $3^{\text {rd }}$ line have in therapy for metastatic NSCLC? Which treatment options exist after failure of TKI in the $1^{\text {st }}$ line? These and other clinically relevant questions in the context of TKIs are discussed in the present comprehensive review. nichtkleinzellige Lungenkarzinome (NSCLC) unterteilt. Ca. 15-25\% aller Lungenkarzinome zählen zu der Gruppe des SCLC [2]. Bis zu 80\% der Fälle sind NSCLC und unterteilen sich im Wesentlichen in das Adenokarzinom, das Plattenepithelkarzinom und das großzellige Lungenkarzinom. Die Prognose für Patienten mit einem NSCLC ist im Vergleich zu anderen Malignomen schlecht. So hat (alle Stadien eingeschlossen) das MammaCa eine 5-Jahres-Überlebensrate von 89\%, das Kolon/Rektum-Ca von $64 \%$, während das Lungen-Ca bei $15 \%$ liegt [3].Selbst beim NSCLC im Tumorstadium I (Tumore bis $3 \mathrm{~cm}$ Größe) und kurativer R0Resektion beträgt die 5-Jahres-Rezidivfreiheit nur 87\% [4]. Eine komplette (R0) Resektion des Karzinoms gibt Hoffnung auf eine Heilung, allerdings liegt die Rezidivquote immer noch unbefriedigend hoch. Kürzlich konnte gezeigt werden, dass im UICC Stadium II nach kurativer Tumorresektion eine adjuvante Chemotherapie das rezidiv- 
freie Überleben auch über 9 Jahre noch signifikant verlängern kann [5]. Welche Patienten aber von dieser Therapie profitieren, ist nicht bekannt. Hier werden in naher Zukunft molekulare Faktoren die Therapieentscheidung nachhaltig beeinflussen, wie von uns kürzlich in einer Übersichtsarbeit zusammengestellt [6]. Zu den aussichtsreichsten Kandidaten gehört das „Excision Repair Cross-Complementing 1“ (ERCC1)-Gen, das für ein Enzym kodiert, das Platin-Addukte erkennen und entfernen kann [7]. Mehrere Studien konnten übereinstimmend zeigen, dass auch in vivo eine hohe Expression von ERCC1 bei verschiedenen Tumorentitäten mit einer Resistenz auf platinhaltige Chemotherapeutika assoziiert ist, während eine niedrige ERCC1-Aktivität mit einem verbesserten Ansprechen und einem längeren Überleben einhergeht $[8,9]$. Diese Erkenntnisse wurden retrospektiv an $1868 \mathrm{Tu}-$ moren der adjuvanten IALT-Studie überprüft und so die prädiktive und gleichzeitig prognostische Bedeutung dieses Markers bestätigt [10].

Bei ca. 40\% der Patienten besteht bei Diagnosestellung ein metastasiertes Krankheitsstadium IV. Für diese Patienten wird neben einer individuellen supportiven Versorgung eine palliative Chemotherapie empfohlen. Hierdurch konnte die mediane Überlebenszeit in Phase-III-Studien über die Jahre deutlich gesteigert werden, von median 7,9 Monaten (im Jahr 2002) [11] bis zu median 11,3 Monaten (im Jahr 2009) [12]. Für die palliative Chemotherapie steht eine Vielzahl an Chemotherapeutika zur Verfügung. Etabliert ist derzeit für die Erstlinientherapie eine platinhaltige Chemotherapie (Cisplatin oder Carboplatin) in Kombination mit einer weiteren Substanz. Als Kombinationspartner sollte ein Taxan, Vinorelbin, Gemcitabin oder Pemetrexed zum Einsatz kommen [13]. Kürzlich konnte gezeigt werden, dass Plattenepithelkarzinome von einer Gemcitabin-haltigen Kombination profitieren, während für die Nicht-Plattenepithelkarzinome Pemetrexed die geeignetere Substanz ist [14]. Der einzige bisher zugelassene Antikörper für die palliative Erstlinientherapie des NSCLC ist Bevacizumab. Dies ist ein „vaskulärer endothelialer Wachstumsfaktor“ (VEGF)-Antikörper, der zusammen mit einer platinbasierten Kombinationschemotherapie beim Nicht-Plattenepithelkarzinom ein mittleres Überleben von bis zu 12,3 Monaten erreicht [15]. Bei Patienten mit schlechtem Allgemeinzustand, ausgeprägten Komorbiditäten und bei älteren Patienten sollte gegebenenfalls an eine Monotherapie gedacht werden [16]. Die Ansprechraten der Kombinationschemotherapie variieren zwischen $11 \%$ und $34 \%[11,17]$. Aufgrund der unterschiedlichen Ansprechrate, der teils nicht unerheblichen Toxizität der eingesetzten Substanzen und der damit häufig verbundenen Minderung der Lebensqualität der Patienten sowie der frühzeitigen Progression der Erkrankung wäre eine individualisierte Therapie mehr als wünschenswert. Dazu müssen validierte präklinische Marker bekannt sein, die den Effekt der Therapie prädizieren [6]. Einen ersten Schritt in diese Richtung stellt nun die Möglichkeit dar, Patienten, die im Tumor eine aktivierende „epidermale Wachstumsfaktor Rezeptor“ (EGF-R)-Mutation aufweisen, zielgerichtet mit einem Tyrosinkinase-Inhibitor (TKI) zu behandeln. Aber auch in weiteren Therapielinien haben die TKIs einen Stellenwert.

Dieser Artikel gibt eine Übersicht über die aktuellen Behandlungsmöglichkeiten mittels TKI. Neben der wissenschaftlichen Grundlage der Therapie mit TKIs und der Vorstellung wichtiger Studien soll die Möglichkeit der Umsetzung und des Einsatzes in der klinischen Praxis diskutiert werden.

\section{Merke}

- Das NSCLC lässt sich vorwiegend in das Adenokarzinom (ADC), das Plattenepithelkarzinom (SCC) und das großzellige Lungenkarzinom (LCC) unterteilen.

- In der palliativen Erstlinientherapie ist - bei ausreichendem Allgemeinzustand - eine platinhaltige Kombinationschemotherapie Standard.

- Als Kombinationspartner ist bei Plattenepithelkarzinomen Gemcitabine und bei Nicht-Plattenepithelkarzinomen Pemetrexed der jeweils anderen Substanz überlegen.

- Der VEGF-Antikörper Bevacizumab ist zusammen mit einer platinhaltigen Kombinationschemotherapie beim Nicht-Plattenepithelkarzinom zugelassen.

- Tyrosinkinase-Inhibitoren (TKIs) erweitern zudem das Armamentarium der zugelassenen Substanzen.

\section{Der EGF-Rezeptor und Mutationen}

$\nabla$

Die Familie der epidermalen Wachstumsfaktoren-Rezeptoren (ErB) besteht aus dem epidermalen Wachstumsfaktor-Rezeptor (engl. epidermal growth factor receptor) EGF-R (= ErbB1) sowie aus Her2/neu (= ErbB2) und ErbB3 und -4 [18]. EGF-R ist ein Glykoprotein, das neben dem epidermalen Wachstumsfaktor (EGF) auch durch eine Reihe weiterer Liganden aktiviert werden kann $[19,20]$. Der EGF-R-Aktivierungsmechanismus verläuft über eine komplexe, multidimensionale, bisher noch nicht vollständig verstandene Signaltransduktions-Kaskade. Es werden dabei verschiedene Bereiche aktiviert, die für den Tumor von existenzieller Bedeutung sind, wie die Unabhängigkeit von Wachstumssignalen, die Resistenz bezüglich Apoptose und wachstumsinhibierenden Impulsen, Angiogenese und Metastasierung. Der EGF-R wird in etwa $80 \%$ der NSCLC exprimiert [21].

Im Mai 2004 haben zwei Arbeitsgruppen aus den USA unabhängig voneinander Mutationen in der Tyrosinkinase-Domäne (Exons 18-23) des EGF-R beschrieben [22,23]. Fast alle Mutationen fanden sich im Bereich der ATP-Bindungstasche, an der auch die TKIs andocken. Weiterhin zeigte sich, dass bestimmte Mutationen mit einer erhöhten Sensitivität bezüglich EGF-R-Inhibition und damit besserer Wirkung der TKIs verbunden sind. So wiesen etwa in einer Untersuchung von Lynch et al. 8 von 9 Respondern (89\%) auf Gefitinib eine EGF-R-Mutation auf, dagegen keiner von 7 Nicht-Respondern ( $<<0,001$ ) [22]. Etwa 90\% aller Mutationen im EGF-R-Gen betreffen nur eine kleine Anzahl von Aminosäuren. So machen Deletionen im Exon 19 (Kodons 746750 ) etwa $45-50 \%$ der Mutationen aus; weitere $35-40 \%$ bestehen in einer Punktmutation im Exon 21, bei der es zum Austausch von Leucin zu Arginin an Position 858 (L858R) kommt [21,24-27]. Etwa 3\% der Mutationen finden sich in Kodon 719 (G719X) im Exon 18, weitere 3\% sind Insertionsmutationen im Exon 20 [28]. All die genannten Mutationen sind zumeist ausschließliche Ereignisse.

Zusätzlich scheint es Unterschiede in der prognostischen Bedeutung der Exon 19- und Exon 21-Mutationen zu geben. So konnten Jackman et al. in einer kürzlich publizierten retrospektiven Arbeit zeigen, dass Exon 19-Deletionen nochmals eine bessere Prognose unter TKI aufweisen als Exon 21-Mutationen [29]. Auch in der großen prospektiven Studie von Rosell et al. zeigte sich ein signifikanter Unterschied zwischen den Mutationstypen [30]. Tumore mit Deletionen im EGF-R-Gen hatten sowohl ein 
besseres progressionsfreies als auch Gesamtüberleben als die mit einer L858R-Mutation.

Kürzlich konnte auch gezeigt werden, dass die Entstehung einer zweiten Mutation im EGF-R (T790M) zu einer Resistenz gegenüber TKI führt, insbesondere dann, wenn die Tumoren zunächst sensibel auf die Substanz waren [31]. Studien, die an Patienten durchgeführt wurden, bei denen eine Rebiopsie nach Progression durchgeführt wurde, zeigten, dass die T790M-Mutation in fast $50 \%$ der Fälle auftrat. Es finden sich aber auch Fälle, bei denen die Mutation bereits bei Therapiebeginn bestand. So stellten Inukai et al. fest, dass 3 von 7 Non-Responder auf eine GefitinibTherapie neben der aktivierenden EGF-R-Mutation eine zweite T790M-Mutation aufwiesen [32]. Dagegen hatte keiner der 19 Responder diese inhibierende Mutation [31]. Interessanterweise kann man T790M-Mutationen im Blut von NSCLC-Patienten nachweisen. In einer zukunftsweisenden Arbeit von Maheswaran et al. wurde zunächst die EGF-R-Mutation aus dem Tumor bestimmt und in 11 von 12 Patienten (92\%) aus zirkulierenden Tumorzellen im Blut der Patienten bestätigt. Darüber hinaus konnte in vielen Fällen die T790M-Mutation nachgewiesen werden. Patienten, die bereits vor Therapie diese Mutation zeigten, hatten ein signifikant schlechteres progressionsfreies Überleben unter der TKI-Therapie (7,7 versus 16,5 Monate; $p<0,001$ ) [33].

Ein weiteres Zielgen, das mit einer Resistenz auf TKI einhergeht, ist der „Hepatozyten Wachstumsfaktor Rezeptor“, der auch als „met-Proto-Onkogen“ (MET) bezeichnet wird [28,34]. Amplifikationen der Rezeptor Tyrosinkinase MET sind für etwa 10-20\% der Resistenzen auf TKI verantwortlich [35]. So konnte gezeigt werden, dass eine zunächst Gefitinib sensitive LungentumorZelllinie durch die Amplifikation des Onkogens MET über den PI3K Signaltransduktionsweg resistent wird [35]. Die Entdeckung von MET scheint für die Zukunft ein interessanter und nützlicher Biomarker für die Prädiktion einer Resistenz auf TKI zu sein.

\section{Merke}

- Aktivierende EGF-R-Mutationen betreffen die ATP-Bindungstasche. Hier binden auch die TKIs.

Exon 19-Deletionen und Exon 21-Punktmutationen machen etwa 95\% der aktivierenden Mutationen aus. Exon 19-Deletionen sind darüber hinaus möglicherweise prognostisch günstiger als Exon 21-Punktmutationen.

T790M ist eine Mutation im EGF-R-Gen, die zur Resistenz gegenüber TKIs führt. Auch Amplifikationen des MET-Gens sind für Resistenzen gegenüber TKI verantwortlich.

\section{TKIs in der palliativen Erstlinientherapie bei EGF-R mutierten Tumoren \\ $\nabla$}

Die reversiblen Tyrosinkinase-Inhibitoren (TKI) Gefitinib und Erlotinib wurden in einer Vielzahl von Studien überprüft. Jedoch führten alle vier randomisierten Studien, die Erlotinib oder Gefitinib in Kombination mit einer Erstlinien-Chemotherapie beim fortgeschrittenen NSCLC einsetzten, zu negativen Ergebnissen [36 - 39]. Allerdings wurden die Studien an einem (in Bezug auf molekulare Faktoren) unselektierten Patientenkollektiv durchgeführt.

Mehrere retrospektive Analysen konnten zeigen, dass Patienten mit aktivierenden EGF-R-Mutationen in der palliativen Erstlinientherapie zu etwa 77\% auf TKI als Monotherapie anspra- chen, im Vergleich zu etwa 10\% ohne EGF-R-Mutation. Und auch das Überleben von Patienten mit Tumoren mit EGF-R-Mutation, die mit TKI therapiert wurden, war deutlich verlängert. Hier wurden Überlebenszeiten von median bis zu 30 Monaten berichtet $[26,40-46]$.

Die IPASS (Iressa Pan-Asia Study), eine randomisierte Phase-IIIStudie, verglich Gefitinib mit einer Kombinations-Chemotherapie, bestehend aus Carboplatin und Paclitaxel, bei 1217 asiatischen Patienten in der Erstlinien-Chemotherapie des fortgeschrittenen NSCLC [47]. Die Patienten wurden eingeschlossen bei Vorliegen bestimmter klinischer Faktoren (Adenokarzinom, Nieraucher oder Exraucher), jedoch erfolgte keine Selektion hinsichtlich molekularer Marker. Die Studie war auf Nichtunterlegenheit von Gefitinib ausgelegt mit einer vordefinierten HR von 1,2. Die Ergebnisse der Studien zeigten aber nicht nur eine Nichtunterlegenheit, sondern sogar eine Überlegenheit von Gefitinib mit einer HR von 0,74 (Konfidenzintervall 0,65-0,85, $\mathrm{p}<0,0001$ ) für das progressionsfreie Überleben. Obwohl sich die Überlebenskurven der beiden Therapie-Arme kreuzten und die ersten sechs Monate die Patienten von der Chemotherapie mehr zu profitieren schienen, sprach das Gesamtergebnis für Gefitinib. Die Überlebensdaten waren in der Publikation noch nicht reif (37\% der Patienten waren verstorben), jedoch ergab sich auch hier eine HR von 0,91 (CI 0,7-1,10) für die Gefitinib-Therapie. Gefitinib zeigte sich der Chemotherapie auch überlegen in Bezug auf Lebensqualität und wies ein verändertes, evtl. günstigeres Toxizitätsprofil auf (weniger Hämatotoxizität, weniger Neurotoxizität, mehr Hautausschlag, mehr Diarrhoe). SubgruppenAnalysen anhand molekularer Marker im Primärtumor konnten bei $30-36 \%$ des Patientenkollektivs durchgeführt werden. Hier zeigte sich, dass bei Vorliegen einer aktivierenden EGF-R-Mutation in Bezug auf progressionsfreies Überleben Gefitinib mit 9,5 Monaten signifikant effektiver als Chemotherapie (mit 6,3 Monaten) war (HR 0,48 CI 0,36-0,64, p<0,0001). Anders verhielt es sich bei den Tumoren, die den EGF-R-Wildtyp auswiesen. Hier war die Chemotherapie (5,5 Monate) der Behandlung mit Gefitinib (1,5 Monate) überlegen (HR 2,85, p < 0,0001). Jedoch lässt die Studie auch Fragen unbeantwortet, wie etwa die ungewöhnlich hohe Fallzahl von EGF-R mutierten Tumoren, der gewählte Endpunkt, progressionsfreies Überleben und die bisher fehlende Überlegenheit im Gesamtüberleben.

Ergebnisse liegen inzwischen auch für zwei weitere asiatische Phase-III-Studien zum Gefitinib vor $[48,49]$. Hier wurde die Substanz ebenfalls wieder mit einem Chemotherapie-Arm, bestehend aus Carboplatin/Paclitaxel bzw. Cisplatin/Docetaxel, verglichen; eingeschlossen wurden hier aber nur Patienten mit aktivierender EGF-R-Mutation im Primärtumor. Die Studien bestätigten die Ergebnisse von IPASS und zeigten ein signifikant besseres progressionsfreies Überleben von 10,4 Monaten (für Gefitinib) versus 5,5 Monaten (für Chemotherapie mit Carbo/Pac) HR $0,357, \mathrm{p}<0,0001$ bzw. HR 0,49 für Gefitinib versus Cis/Doc $\mathrm{p}<0,0001$. Aufgrund der gezeigten Datenlage wurde Gefitinib im Juli 2009 zur Behandlung von EGF-R mutierten fortgeschrittenen NSCLC in allen Therapielinien zugelassen.

Alle bisher aufgeführten Studien wurden an asiatischen Patienten durchgeführt. Für europäische bzw. kaukasische Patienten liegen bis dato nur wenige prospektive Ergebnisse für eine EGFR-Mutation als prädiktiver Marker für das Ansprechen und/oder Überlebensvorteil für TKI vor. In einer kleinen prospektiven Phase-II-Studie wurden 34 kaukasische Patienten, die eine aktivierende EGF-R-Mutation aufwiesen, mit Gefitinib in der palliativen Erstlinientherapie behandelt [50]. Mit einem Ansprechen 
von 55\% und einem progressionsfreien Überleben von 9,2 Monaten sind diese Daten mit denen asiatischer Patienten vergleichbar. Die bisher größte prospektive Studie europäischer Patienten wurde in Spanien durchgeführt [30]. Rosell et al. untersuchten 2.105 NSCLC-Patienten auf das Vorliegen von aktivierenden EGFR-Mutationen im Tumor. 360 (16,6\%) wiesen eine Mutation auf; 217 von ihnen wurden daraufhin in der palliativen Erst- oder Zweitlinie mit dem TKI Erlotinib behandelt. 70\% zeigten ein Ansprechen auf die Therapie, $12 \%$ sogar eine komplette Remission. Das mediane progressionsfreie Überleben betrug 14 Monate, das mediane Gesamtüberleben 27 Monate. Auch diese Studie belegt die Effektivität der TKI-Therapie.

Dass auch alte Patienten bzw. Patienten in einem schlechten Allgemeinzustand, die nicht mehr chemotherapiefähig sind, von der Therapie mit einem TKI profitieren können, zeigten Inoue et al. kürzlich in einer kleinen Studie [51]. 30 asiatische Patienten mit EGF-R mutierten Tumoren, davon 22 mit einem ECOG-PS von 3 oder 4 erhielten Gefitinib in der palliativen Erstlinientherapie. Eine Tumorkontrolle wiesen $90 \%$ dieser Patienten auf. Weitaus bedeutsamer war, dass von den o.g. 22 Patienten $68 \%$ sich im Allgemeinzustand von $\geq$ PS 3 auf $\leq$ PS 1 verbesserten. Mit einem Gesamtüberleben in dieser Gruppe von median 17,8 Monaten sprechen manche Kommentare von einem „Lazarus-Phänomen“.

Merke

- Tumore mit aktivierender Mutation im EGF-R sprechen in hohem Maße auf eine palliative Erstlinientherapie mit TKI an. - Das bessere Ansprechen unter TKI manifestiert sich in diesen Fällen auch in einem besseren krankheitsfreien Überleben; das Gesamtüberleben war bisher nicht signifikant verbessert.

- Lebensqualitätsanalysen sprechen bei nachgewiesener, aktivierender EGF-R-Mutation ebenfalls für die Therapie mit TKI. - Auch Patienten in schlechtem Allgemeinzustand können von der TKI-Therapie profitieren, wenn eine aktivierende EGF-RMutation vorliegt.

Die Therapie mit dem TKI Gefitinib ist seit Juli 2009 zur Behandlung des fortgeschrittenen NSCLC mit aktivierender Mutation im EGF-R-Gen in allen Therapielinien zugelassen.

\section{Wer soll getestet werden?}

$\nabla$

Von der Erstlinientherapie mit einem TKI profitieren fast ausschließlich Patienten, die eine aktivierende EGF-R-Mutation in ihrem Tumor aufweisen. Daher sollten aus medizinischer Sicht alle Patienten auf die EGF-R-Mutation hin getestet werden, für die im Fall einer nachgewiesenen aktivierenden EGF-R-Mutation eine Therapie mit einem TKI in Frage kommt. Die anfallenden Kosten von derzeit 300 - 500 Euro für die Mutationsbestimmung werden jedoch in der Primärdiagnostik des NSCLC derzeit nicht abgebildet. Daher ist in der klinischen Praxis außerhalb von klinischen Studien die Testung aller Patienten finanziell unmöglich und klinische Parameter müssen herangezogen werden, um eine Vorselektion zu treffen.

In einem weitgehend unselektierten spanischen Patientenkollektiv (nur Plattenepithelkarzinome waren ausgeschlossen) von 2105 Patienten lag die Mutationshäufigkeit bei 16,6\% [30]. Bestimmte Subgruppen hatten jedoch deutlich höhere Mutationsfrequenzen: so lag die Mutationshäufigkeit bei Frauen bei 30\% (versus 8,2\% für Männer). Auch der Raucherstatus schlug sich in unterschiedlichen Mutationsraten nieder; während Tumoren von
Nierauchern zu 37,7\% Mutationen aufwiesen, so waren Exraucher zu 9,5\% und Raucher nur zu 5,8\% mutiert. Interessanterweise war auch das Alter von Bedeutung; während 22,1\% der über 69-jährigen Patienten eine Mutation aufwiesen, waren es in der Altersgruppe $<59$ Jahre nur $13,9 \%$. Sequist et al. testeten in einer prospektiven Phase-II-Studie Patienten, die mindestens eines der folgenden Kriterien aufwiesen: weibliches Geschlecht, Adenokarzinome jeglichen Subtyps, asiatische Abstammung, Nie- oder Nichtraucher (<100 Zigaretten) [50]. Von 98 (davon 93 Nichtasiaten) in die Studie eingeschlossenen und „gescreenten“ Patienten hatten $33 \%$ eine aktivierende EGF-R-Mutation. Für Deutschland sind in den nächsten Jahren Daten über die Mutationshäufigkeit zu erwarten, die in einer groß angelegten epidemiologische Studie (REASON) derzeit erhoben werden.

\section{Erhaltungstherapie (Maintenance)}

Derzeit empfehlen die meisten Leitlinien 4-6 Zyklen einer kombinierten Erstlinien-Chemotherapie [1]. Aktuell richtet sich das wissenschaftliche Interesse auf eine Verlängerung der Erstlinien-Behandlung, da mehrere prospektive Studien einen möglichen Benefit dieser Strategie zeigen konnten. Grundsätzlich kann dies dadurch erreicht werden, dass ohne klinische Anzeichen eines Progresses eine oder mehrere Substanzen weitergeführt werden oder mit einer neuen Substanz nahtlos weiter therapiert wird. Ob diese Strategie dann, insbesondere wenn eine neue Substanz ins Spiel gebracht wird, als Maintenance, sequenzielle Therapie oder frühe Zweitlinientherapie bezeichnet werden sollte, ist ein semantisches Problem.

Im SATURN-Trial, einer prospektiv randomisierten Phase-III-Studie, wurden Patienten, die nach 4 Zyklen einer platinbasierten Chemotherapie nicht progredient waren, entweder mit dem TKI Erlotinib oder Placebo weitertherapiert [52]. Das Patientenkollektiv wurde nicht nach klinischen oder molekularen Faktoren selektiert; der EGF-R-Status war jedoch ein Stratifizierungsmerkmal. Der primäre Endpunkt, das progressionsfreie Überleben, wurde erreicht und zeigte einen signifikanten Vorteil für diejenigen Patienten, die Erlotinib erhalten hatten (HR 0,71 CI 0,620,82, p<0,0001). Auch das Gesamtüberleben (ab Randomisierung nach 4 Zyklen Chemotherapie) war unter Erlotinib Maintenance signifikant $(\mathrm{p}=0,009)$ verlängert, wenngleich die klinische Relevanz (12 Monate versus 11 Monate im Placebo-Arm) zu diskutieren ist. An 49\% der Tumore konnte der EGF-R-Mutationsstatus überprüft werden. Es zeigte sich, dass Patienten mit aktivierender EGF-R-Mutation ein deutlich besseres PFS hatten, aber auch Wildtyp-Patienten profitierten von der Therapie (HR 0,10 für EGF-R+ versus 0,78 für WT). Im Gesamtüberleben zeigte sich kein signifikanter Unterschied zwischen EGF-R mutierten und nicht mutierten Tumoren, jedoch hatten auch im Placebo-Arm 67\% der Patienten mit EGF-R-Mutation Erlotinib in der weiteren Behandlung erhalten.

Erlotinib wurde auch in einer weiteren prospektiv randomisierten Studie, dem ATLAS-Trial, als Maintenance weitergeführt [53]. In diesem Studiendesign wurde nach Tumorkontrolle auf eine palliative platinhaltige Kombinationstherapie mit Bevacizumab entweder nur der VEGF-Antikörper oder Bevacizumab und Erlotinib als Maintenance weitergeführt. Auch hier wurde nicht nach EGF-R-Mutationsstatus selektiert. Nach der zweiten Interimsanalyse wurde die Studie gestoppt, weil der primäre Endpunkt, das progressionsfreie Überleben, erreicht worden war. Mit einem PFS von 4,76 Monaten war die Kombinations-Maintenance-The- 
rapie signifikant der alleinigen Bevacizumab Maintenance $(3,75$ Monate) überlegen (HR 0,722, p=0,0012). Subgruppenanalysen in Bezug auf molekulare Marker wurden bisher noch nicht berichtet.

Diese Ergebnisse müssen mit Zurückhaltung bewertet werden. Insgesamt hat nur eine Minderheit von Patienten (16\% in der SATURN-Studie und 39\% in der ATLAS-Studie) Erlotinib im weiteren Behandlungsverlauf erhalten. In wie weit sich die Gesamtüberlebensdaten geändert hätten, wenn ein Crossover nach Progress der Maintenance-Therapie geplant worden wäre, bleibt Spekulation. Aus einer anderen Maintenance-Studie, bei der die Therapie mit Docetaxel weitergeführt wurde, weiß man, dass alle Patienten, die die Substanz erhalten haben - also entweder als Maintenance- oder als Zweitlinientherapie - das gleiche Gesamtüberleben (im Median 12,5 Monate) hatten [54].

Derzeit ist noch nicht abschließend geklärt, welches Patientenkollektiv am meisten von der Maintenance-Therapie profitiert und welche Patienten - auch aus Sicht der Toxizität und Lebensqualität - bis zum Progress der Erkrankung eine Therapiepause haben sollten. Aus Daten einer retrospektiven Analyse zur Vergleichsstudie von Pemetrexed und Docetaxel in der Zweitlinie weiß man allerdings, dass das Ansprechen auf die Erstlinientherapie eine wichtige Rolle spielt [55]. Während Patienten mit einem Ansprechen auf die Erstlinientherapie ein medianes Gesamtüberleben von 15,8 Monaten aufwiesen, lag es bei Patienten mit stabiler Krankheitssituation bei 10,5 Monaten und bei Progress auf die Erstlinie sogar nur bei 4,6 Monaten. Das Ansprechen war auch in der multivariaten Analyse ein unabhängiger Prognosefaktor $(\mathrm{p}<0,001)$.

Ganz aktuelle Daten zeigen, dass in der o.g. SATURN-Studie die Patienten mit stabiler Erkrankungssituation nach der Erstlinientherapie einen signifikanten Vorteil von der Erhaltungstherapie mit Erlotinib hatten $[52,56,57]$. So lag in dieser Gruppe das Gesamtüberleben bei 11,9 Monaten (im Vergleich zu Placebo: 9,6 Monaten; HR 0,72; p=0,0019). Die Erhaltungstherapie wurde daher im Mai 2010 in Deutschland bei dieser Patientengruppe zugelassen.

\section{Merke}

- Die Erhaltungstherapie (Maintenance) stellt eine Option zur Weiterführung einer Erstlinien-Chemotherapie dar.

- Die Phase-III-Studien zur Erhaltungstherapie beim NSCLC haben alle das Hauptzielkriterium, eine Verbesserung des progressionsfreien Überlebens, erreicht.

- Leider haben im Kontrollarm nicht alle Patienten die Prüfsubstanz im weiteren Verlauf der Erkrankung erhalten, sodass die Aussagen zum Gesamtüberleben mit Vorsicht zu interpretieren sind.

- Die Erhaltungstherapie mit dem TKI Erlotinib zeigte kürzlich sowohl für das progressionsfreie als auch für das Gesamtüberleben einen signifikanten Benefit bei Patienten, bei denen die Erstlinientherapie eine stabile Krankheitssituation bewirkte. Dieses Therapiekonzept wurde daher im Mai 2010 zugelassen.

\section{Zweit- und Drittlinientherapie}

\section{$\nabla$}

Zwei große Phase-III-Studien evaluierten Erlotinib (BR.21-Studie) oder Gefitinib (ISEL-Studie) mit Placebo und beide zeigten eine signifikante Verbesserung der Zeit bis zur Tumorprogression um etwa 0,4 Monate $[58,59]$. Auch das Gesamtüberleben war in der BR.21-Studie im TKI-Arm signifikant länger (6,7 versus 4,7 Monate, $\mathrm{p}<0,001)$; ein Trend zur Verbesserung des Überlebens unter TKI zeigte sich in ISEL (5,6 versus 5,1 Monate, $\mathrm{p}=0,087$ ). Klinisch in der Zweit- und Drittlinie bedeutsamer als das Überleben ist der Einfluss auf die Tumorsymptome und die Lebensqualität. Hier verbesserte sich im BR.21-Trial die Zeit bis zur Verschlechterung von tumorbedingten Symptomen (Husten, Schmerz, Dyspnoe) signifikant [60], während es in ISEL unter TKI-Behandlung bei 27\% der Patienten zu einer Verbesserung der krankheitsbedingten Symptome kam (versus 22\% im Placebo-Arm). Darüber hinaus verbesserte sich die Lebensqualität signifikant $(p=0,019)$. Retrospektive Daten zum EGF-R-Mutationsstatus liegen nur für BR.21 vor. Hier zeigte sich in der multivariaten Analyse von 204 Tumoren kein Einfluss des Mutationsstatus auf das Überleben (Mut+ HR:0,55 versus WT HR:0,74, p=0,47) [61,62].

Drei große randomisierte Studien verglichen Gefitinib mit Docetaxel, das als einer der Standards in der Zweitlinientherapie des NSCLC gilt [63-65]. In einer davon zeigte sich eine signifikante Verbesserung des Ansprechens unter Gefitinib (22,5\% versus $12,8 \%$ unter Docetaxel, $\mathrm{p}=0,009$ ); die beiden anderen Untersuchungen konnten das nicht belegen. Auch in der Zeit bis zur Tumorprogression (TTP) und im Gesamtüberleben zeigten sich in allen drei Studien keine signifikanten Unterschiede. Insbesondere die große INTEREST-Studie konnte zeigen, dass Gefitinib nicht inferior zu Docetaxel war (HR 1,02 CI 0,905-1,15) [64]. Von besonderer Bedeutung ist diese Studie, weil hier die Anzahl an Patienten, die eine effektive Drittlinientherapie erhielten, in beiden Armen ausgewogen und somit auch das Gesamtüberleben vergleichbar war.

Im Gegensatz zur Erstlinientherapie scheint für den Einsatz von TKI in weiteren Therapielinien die Bedeutung des EGF-R-Mutationsstatus in den Hintergrund zu rücken. Zwar haben Patienten mit EGF-R-Mutation ein signifikant besseres progressionsfreies Überleben, wie sich etwa in molekulargenetischen Auswertungen zur TRUST-Studie zeigt, aber auch Patienten mit EGF-R-Wildtyp profitieren offensichtlich von der Therapie mit Erlotinib [66]. In dieser Studie wurden 311 Patienten mit metastasiertem NSCLC behandelt, davon $40 \%$ in der Zweit- und $37 \%$ in der Drittlinientherapie. EGF-R-Mutationen fanden sich in 6 von 92 untersuchten Tumoren (7\%). Diese Patienten hatten sowohl ein signifikant verbessertes progressionsfreies Überleben (HR 0,31) als auch ein verbessertes Gesamtüberleben (HR 0,33). Jedoch wiesen insgesamt 66\% der Patienten einen klinischen Benefit in Form einer Tumorkontrolle auf. Dieser positive Effekt zeigte sich noch deutlicher in einer retrospektiven Auswertung von 301 Patienten, die länger als 12 Monate mit dem TKI Erlotinib behandelt werden konnten [67]. Zwar wurden hier keine EGF-R-Mutationsanalysen durchgeführt, doch die hohe Zahl an männlichen Patienten (43\%), an Patienten mit positivem Raucherstatus (14\% Raucher, 34\% Exraucher) sowie mit Plattenepithelkarzinom (16\%) ist nicht vergleichbar mit dem „klassischen“ EGF-R-Mutationskollektiv, bei dem weibliche Patienten, Nichtraucher und Adenokarzinome dominieren. Interessanterweise zeigte sich bei der Mehrzahl der Patienten (53\%) kein Ansprechen, sondern lediglich eine stabile Krankheitssituation. Dennoch konnten immerhin 25\% der Patienten über 2 Jahre mit dem TKI behandelt werden. 
Insgesamt haben Raucher einen geringeren Benefit von TKI als Nichtraucher und Exraucher. In einer Analyse der BR.21-Studie zeigte sich, dass Raucher ein signifikant geringeres Ansprechen auf die Therapie mit Erlotinib haben, jedoch auch weniger Toxizität [51]. Es wurde daher vermutet, dass die Plasmaspiegel des Medikaments bei Rauchern zu niedrig sind, und eine Dosis-Eskalations-Studie durchgeführt [68]. Die maximal tolerierte Dosis lag bei Rauchern bei $300 \mathrm{mg}$; dies entspricht dem doppelten der herkömmlich eingesetzten Dosierung. Im zweiten Teil der Studie hatten Raucher, die mit 300 mg behandelt wurden, die gleiche Toxizität wie Nichtraucher mit $150 \mathrm{mg}$. Inwieweit die erhöhte Dosierung bei Rauchern auch zu einer Verbesserung der Effektivität führt, wird in einer Phase-III-Studie (CURRENTS) überprüft. Ein direkter Vergleich zwischen den beiden TKI in der palliativen Zweitlinientherapie wurde von Uhm et al. durchgeführt [69]. In einer kleinen randomisierten Phase-II-Studie an einem in Bezug auf klinische Faktoren bzw. EGF-R-Mutation selektierten Patientenkollektiv erhielten 96 asiatische Patienten entweder Erlotinib oder Gefitinib. Hauptzielkriterium dieser Studie war das Ansprechen auf die Therapie. Es zeigten sich keine signifikanten Unterschiede zwischen beiden TKI, weder im Ansprechen noch in der Tumorkontrolle. Allerdings traten unter Erlotinib signifikant mehr Nebenwirkungen in Form von Rash $(p=0,003)$ sowie Fatigue ( $p=0,027)$ auf. Das mediane Gesamtüberleben der Gesamtgruppe betrug 20,4 Monate.

\section{Merke}

- Auch in der Zweit- und Drittlinientherapie haben TKI einen wichtigen Stellenwert.

- Für den Einsatz von Gefitinib ist auch hier der Nachweis einer aktivierenden EGF-R-Mutation zulassungsrechtliche Voraussetzung.

- Eine EGF-R-Mutationstestung scheint hier jedoch nicht essenziell zu sein, weil Studien mit Erlotinib gezeigt haben, dass auch Patienten mit EGF-R-Wildtyp einen Benefit haben können.

Raucher benötigen evtl. eine höhere Konzentration des TKI Erlotinib. Dies wird aktuell in einer Phase-III-Studie überprüft.

\section{Therapie nach Progress auf TKI \\ $\nabla$}

Selbst bei Patienten, die in ihrem Tumor eine aktivierende EGF-RMutation aufweisen und ein gutes Ansprechen auf die TKI-Therapie haben, kommt es nach einer gewissen Zeit zum Progress der Erkrankung. Nach Absetzen der TKI-Therapie nimmt das Tumorwachstum dann häufig sehr schnell zu. Theoretisch gibt es nach TKI-Versagen außerhalb klinischer Studien mehrere Möglichkeiten der Zweitlinientherapie: a) mit einem anderen TKI (z.B. mit Erlotinib nach Gefitinib-Versagen), b) mit einer Kombination aus TKI und Chemotherapie, c) mit Chemotherapie. Für alle diese Möglichkeiten gibt es mittlerweile publizierte Kasuistiken oder kleine Fallserien.

a) Die Weiterführung mit einem anderen TKI nach Versagen des TKI in der Erstlinientherapie scheint keine geeignete Strategie zu sein. Zwar gibt es einzelne Fallkasuistiken, die einen klinischen Benefit beschreiben, in zwei größeren retrospektiven Analysen zeigte sich jedoch nur ein minimaler Effekt [70,71]. In der Untersuchung von Costa et al. beispielsweise erhielten 18 Patienten nach initialem Ansprechen auf den TKI Gefitinib bei Progress den TKI Erlotinib als Zweitlinientherapie. Bei 78\% kam es zu einem Progress. Ursächlich war zumeist eine sekundäre T790M-Mutation, gegen die auch Erlotinib nichts bewirken konnte. Lediglich vier Patienten hatten eine Tumorkontrolle (davon eine partielle Remission) mit einem progressionsfreien Überleben zwischen 3,7 bis 6 Monaten. In einer weiteren Studie von Wu et al. war der Effekt sogar noch schlechter. Nur 4 von 72 Patienten (5,6\%) zeigten ein Ansprechen auf eine Erlotinib Zweitlinientherapie nach Gefitinib Erstlinie. Von diesen vier Patienten hatten interessanterweise 3 kein Ansprechen auf Gefitinib gehabt, obwohl eine EGF-RMutation vorlag.

b) Eine Kombination aus Gefitinib und Paclitaxel nach Gefitinib haben kürzlich Shukuya et al. berichtet [72]. 15 asiatische Patienten mit Adenokarzinom wurden behandelt und retrospektiv analysiert. Bei allen Patienten war bereits mindestens eine Chemotherapie vorausgegangen und alle hatten auf Gefitinib ein initiales Ansprechen gezeigt. Durch Hinzufügen von Paclitaxel kam es in $80 \%$ zu einer erneuten Tumorkontrolle (davon zwei Patienten mit partieller Remission). Das mediane progressionsfreie Intervall betrug 4,3 Monate, das 1-JahresÜberleben 25\%. Die Therapie wurde gut vertragen; Fatigue, Anorexie und Neutropenie (jeweils CTC Grad 3) traten bei jeweils einem Patienten auf.

c) Die Therapieoption mit Chemotherapie nach TKI-Versagen wurde bisher an der größten Patientenzahl retrospektiv untersucht [71]. Insgesamt 195 asiatische Patienten haben nach Versagen einer Therapie mit dem TKI Gefitinib eine Zweitlinientherapie erhalten, davon 123 eine Chemotherapie. Untersucht wurden die Substanzen Gemcitabine, Taxan und Vinorelbin, entweder als Monotherapie oder in Kombination mit einem Platinderivat. Es zeigte sich, dass die platinhaltige Kombinationstherapie ein signifikant besseres Ansprechen erzielte als die Monotherapie (27\% versus 7\%; $p=0,015$ ). Als Kombinationspartner waren die Taxane mit 50\% Ansprechrate (6 von 12 Patienten) am besten geeignet. Auch in Bezug auf das mediane Gesamtüberleben waren platinhaltige Kombinationen der Monotherapie signifikant überlegen (21,7 versus 10,1 Monate; $\mathrm{p}=0,011$ ). Der Kombinationspartner spielte für diesen Effektivitätsparameter offensichtlich keine entscheidende Rolle. Interessant und unerwartet war, dass Patienten mit EGF-R mutiertem Tumor ein (wenn auch nicht signifikant) schlechteres Ansprechen (15 versus 27\%) und ein kürzeres Überleben (10,3 versus 12,1 Monate) auf die Chemotherapie Zweitlinie hatten als solche mit Wildtyp.

Nach Gefitinib Erstlinie und platinhaltiger Zweitlinien-Chemotherapie könnte in der palliativen Drittlinie möglicherweise eine Reinduktion mit Gefitinib versucht werden. Einzelne Kasuistiken und kleinere retrospektive Fallserien zeigen übereinstimmend einen klinischen Benefit dieses therapeutischen Konzepts mit einer erneuten progressionsfreien Zeit von zum Teil mehr als 7 Monaten [73-76]. Es wird vermutet, dass einige Tumore durch die Chemotherapie wieder sensibel auf den TKI werden könnten, weil sich das Verhältnis von resistenten und sensiblen Klonen verschiebt. Denkbar sind auch genetische Veränderungen im EGF-R-Gen oder anderen für die Regulation wichtiger Gene, die im weiteren Tumorverlauf entstehen. Um diesen interessanten Aspekt weiter zu erforschen, müssten prospektive Studien durchgeführt werden, die mittels Rebiopsie am Tumor vor der Reinduktionstherapie ein aktuelles Bild der genetischen Veränderungen zulassen. 


\section{Merke}

- Nach Progress auf eine Erstlinientherapie mit TKI stehen mehrere Möglichkeiten der Zweitlinientherapie zur Verfügung. Allerdings sind die bisherigen Daten alle retrospektiv gewonnen und müssen prospektiv überprüft werden.

Die Fortführung mit einem anderen TKI scheint in aller Regel keinen Sinn zu machen. Dagegen ist möglicherweise eine Kombinationschemotherapie mit einem Platin und einem Taxan zielführend in Bezug auf erneutes Ansprechen und krankheitsfreies Überleben.

Als Drittlinie kann evtl. eine erneute Therapie mit einem TKI versucht werden.

\section{Klinische Empfehlung und Ausblick \\ $\nabla$}

Gegen den EGF-R gerichtete TKI erweitern das Armamentarium der medikamentösen Tumortherapie des fortgeschrittenen NSCLC. Insbesondere Patienten, die eine aktivierende Mutation des EGF-R in ihrem Tumor aufweisen, profitieren von einer Erstoder Zweitlinientherapie mit einem TKI, wobei für die Erstlinientherapie derzeit nur Gefitinib zugelassen ist. Patienten, die in ihren Tumoren den Wildtyp-Rezeptor exprimieren, sollten dagegen in der Erstlinie mit einer Kombinationschemotherapie behandelt werden. Für die Zweit- und Drittlinie sowie für eine MaintenanceTherapie gibt es Daten für die Therapie mit dem TKI Erlotinib. Hier ist eine EGF-R-Mutationsbestimmung vor Therapieeinleitung nach heutigen Erkenntnissen nicht notwendig. Eine neue Klasse an dualen, irreversiblen EGFR/HER2-Inhibitoren (z. B. BIBW 2992, HKI-272) wird derzeit in mehreren klinischen Studien getestet. Präklinische Daten konnten zeigen, dass diese irreversiblen TKIs in präklinischen Modellen wirksam sind und Resistenzen weniger häufig sind [77 - 79]. Anders als die reversiblen EGF-R-TKIs Erlotinib und Gefitinib binden die irreversiblen TKIs an der Kinase-Domäne kovalent, was den Vorteil hat, dass sie auch Resistenzen, wie etwa die T790M-Mutation, umgehen können. Aber auch für die MET-Gen-Amplifikationen, die zur Resistenz führen, sind Inhibitoren in der klinischen Entwicklung [80].

Zusammenfassend haben die TKI einen wichtigen Stellenwert in der palliativen Therapie des fortgeschrittenen NSCLC. Auch in der adjuvanten Situation werden die TKI derzeit überprüft. In der RADIANT-Studie beispielsweise wird Erlotinib in den Stadien IB - IIIA bei EGF-R mutierten Tumoren gegen Placebo getestet [81]; Ergebnisse zur Effektivität werden hier aber erst in ein paar Jahren erwartet.

\section{Interessenkonflikt \\ $\nabla$}

W. Brückl erhielt Honorar für Vorträge und Beratertätigkeit: Roche, Astra Zeneca

G. Wiest erhielt Honorar für Vorträge und Beratertätigkeit: Roche, Astra Zeneca

J. Ficker erhielt Honorar für Vorträge und Beratertätigkeit:

Roche, Astra Zeneca

\section{Literatur}

1 Goeckenjan G, Sitter H, Thomas M et al. Prävention, Diagnostik, Therapie und Nachsorge des Lungenkarzinoms. Interdisziplinäre S3-Leitlinie der Deutschen Gesellschaft für Pneumologie und Beatmungsmedizin und der Deutschen Krebsgesellschaft. Pneumologie 2010; 64: e1e124

2 Jackman DM, Johnson BE. Small-cell lung cancer. Lancet 2005; 366: $1385-1396$

3 Jemal A, Siegel R, Ward E et al. Cancer statistics, 2009. CA Cancer J Clin 2009; 59: 225-249

4 Maeda R, Yoshida J, Ishii G et al. Long-term survival and risk factors for recurrence in stage I non-small cell lung cancer patients with tumors up to $3 \mathrm{~cm}$ in maximum dimension. Chest 2010; Online-Publikation DOI: $10.1378 /$ chest.09-3046]

5 Butts CA, Ding K, Seymour L et al. Randomized phase III trial of vinorelbine plus cisplatin compared with observation in completely resected stage IB and II non-small-cell lung cancer: updated survival analysis of JBR-10. J Clin Oncol 2010; 28: 29-34

6 Zirlik S, Lampert S, Hahn EG et al. An approach to individualised therapy of non-small cell lung carcinoma (NSCLC) - relevance of molecular predictive and prognostic factors. Pneumologie 2007; 61: 731 - 738

7 Cecere F, Bria E, Rosell $R$. DNA repair by ERCC1 in non-small-cell lung cancer. N Engl J Med 2006; 355: 2590 - 2591; author reply 2591

8 Dabholkar M, Vionnet J, Bostick-Bruton F et al. Messenger RNA levels of XPAC and ERCC1 in ovarian cancer tissue correlate with response to platinum-based chemotherapy. J Clin Invest 1994; 94: 703-708

9 Metzger R, Leichman CG, Danenberg KD et al. ERCC1 mRNA levels complement thymidylate synthase mRNA levels in predicting response and survival for gastric cancer patients receiving combination cisplatin and fluorouracil chemotherapy. J Clin Oncol 1998; 16: $309-316$

10 Olaussen KA, Dunant A, Fouret $P$ et al. DNA repair by ERCC1 in nonsmall-cell lung cancer and cisplatin-based adjuvant chemotherapy. $\mathrm{N}$ Engl J Med 2006; 355: 983 - 991

11 Schiller JH, Harrington D, Belani CP et al. Comparison of four chemotherapy regimens for advanced non-small-cell lung cancer. N Engl J Med 2002; 346: $92-98$

12 Pirker R, Pereira JR, Szczesna A et al. Cetuximab plus chemotherapy in patients with advanced non-small-cell lung cancer (FLEX): an open-label randomised phase III trial. Lancet 2009; 373: 1525-1531

13 Gridelli C, Ardizzoni A, Douillard JY et al. Recent issues in first-line treatment of advanced non-small-cell lung cancer: Results of an International Expert Panel Meeting of the Italian Association of Thoracic Oncology. Lung Cancer 2010; 68: 319-331

14 Scagliotti GV, Parikh P, Pawel J von et al. Phase III study comparing cisplatin plus gemcitabine with cisplatin plus pemetrexed in chemotherapy-naive patients with advanced-stage non-small-cell lung cancer. J Clin Oncol 2008; 26: 3543 - 3551

15 Sandler A, Gray R, Perry MC et al. Paclitaxel-carboplatin alone or with bevacizumab for non-small-cell lung cancer. N Engl J Med 2006; 355: $2542-2550$

16 Gridelli $C$. The ELVIS trial: a phase III study of single-agent vinorelbine as first-line treatment in elderly patients with advanced non-small cell lung cancer. Elderly Lung Cancer Vinorelbine Italian Study. Oncologist 2001; 6 Suppl 1: $4-7$

17 Reck M, Pawel J von, Zatloukal P et al. Phase III trial of cisplatin plus gemcitabine with either placebo or bevacizumab as first-line therapy for nonsquamous non-small-cell lung cancer: AVAil. J Clin Oncol 2009; 27: $1227-1234$

18 Wells A. EGF receptor. Int J Biochem Cell Biol 1999; 31: 637 - 643

19 Brueckl WM, Schoeberl A, Wirtz RM et al. Increased vascular-endothelial growth factor (VEGF) tumor expression and response to epidermal growth factor receptor (EGF-R) inhibitor erlotinib in non-small cell lung cancer (NSCLC). J Thorac Oncol 2008; 3: 314-316

20 Gamou S, Hunts J, Harigai H et al. Molecular evidence for the lack of epidermal growth factor receptor gene expression in small cell lung carcinoma cells. Cancer Res 1987; 47: 2668-2673

21 Shigematsu H, Lin L, Takahashi T et al. Clinical and biological features associated with epidermal growth factor receptor gene mutations in lung cancers. J Natl Cancer Inst 2005; 97: 339-346

22 Lynch TJ, Bell DW, Sordella $R$ et al. Activating mutations in the epidermal growth factor receptor underlying responsiveness of non-smallcell lung cancer to gefitinib. N Engl J Med 2004; 350: 2129-2139

23 Paez JG, Janne PA, Lee JC et al. EGFR mutations in lung cancer: correlation with clinical response to gefitinib therapy. Science $2004 ; 304$ : $1497-1500$ 
24 Kosaka T, Yatabe Y, Endoh $\mathrm{H}$ et al. Mutations of the epidermal growth factor receptor gene in lung cancer: biological and clinical implications. Cancer Res 2004; 64: 8919-8923

25 Marchetti A, Martella C, Felicioni L et al. EGFR mutations in non-smallcell lung cancer: analysis of a large series of cases and development of a rapid and sensitive method for diagnostic screening with potential implications on pharmacologic treatment. J Clin Oncol 2005; 23: 857865

26 Mitsudomi T, Kosaka T, Endoh $H$ et al. Mutations of the epidermal growth factor receptor gene predict prolonged survival after gefitinib treatment in patients with non-small-cell lung cancer with postoperative recurrence. J Clin Oncol 2005; 23: 2513-2520

27 Tomida S, Koshikawa K, Yatabe Y et al. Gene expression-based, individualized outcome prediction for surgically treated lung cancer patients. Oncogene 2004; 23: 5360 - 5370

28 Gazdar AF. Activating and resistance mutations of EGFR in non-smallcell lung cancer: role in clinical response to EGFR tyrosine kinase inhibitors. Oncogene 2009; 28 Suppl 1: S24-S31

29 Jackman DM, Miller VA, Cioffredi LA et al. Impact of epidermal growth factor receptor and KRAS mutations on clinical outcomes in previously untreated non-small cell lung cancer patients: results of an online tumor registry of clinical trials. Clin Cancer Res 2009; 15: 5267-5273

30 Rosell R, Moran T, Queralt C et al. Screening for epidermal growth factor receptor mutations in lung cancer. N Engl J Med 2009; 361: 958 - 967

31 Kobayashi S, Boggon TJ, Dayaram T et al. EGFR mutation and resistance of non-small-cell lung cancer to gefitinib. N Engl J Med 2005; 352: $786-792$

32 Inukai $M$, Toyooka S, Ito $S$ et al. Presence of epidermal growth factor receptor gene T790M mutation as a minor clone in non-small cell lung cancer. Cancer Res 2006; 66: 7854-7858

33 Maheswaran S, Sequist LV, Nagrath $S$ et al. Detection of mutations in EGFR in circulating lung-cancer cells. N Engl J Med 2008; 359: 366 377

34 Hammerman PS, Janne PA, Johnson BE. Resistance to Epidermal Growth Factor Receptor Tyrosine Kinase Inhibitors in Non-Small Cell Lung Cancer. Clin Cancer Res 2009; 15: 7502 - 7509

35 Engelman JA, Janne PA. Mechanisms of acquired resistance to epidermal growth factor receptor tyrosine kinase inhibitors in non-small cell lung cancer. Clin Cancer Res 2008; 14: 2895 - 2899

36 Gatzemeier U, Pluzanska A, Szczesna A et al. Phase III study of erlotinib in combination with cisplatin and gemcitabine in advanced non-smallcell lung cancer: the Tarceva Lung Cancer Investigation Trial. J Clin Oncol 2007; 25: 1545 - 1552

37 Giaccone G, Herbst RS, Manegold C et al. Gefitinib in combination with gemcitabine and cisplatin in advanced non-small-cell lung cancer: a phase III trial - INTACT 1. J Clin Oncol 2004; 22: 777 - 784

38 Herbst RS, Giaccone G, Schiller JH et al. Gefitinib in combination with paclitaxel and carboplatin in advanced non-small-cell lung cancer: a phase III trial - INTACT 2. J Clin Oncol 2004; 22: 785 - 794

39 Herbst RS, Prager D, Hermann R et al. TRIBUTE: a phase III trial of erlotinib hydrochloride (OSI-774) combined with carboplatin and paclitaxel chemotherapy in advanced non-small-cell lung cancer. J Clin Oncol 2005; 23: $5892-5899$

40 Cappuzzo F, Hirsch FR, Rossi E et al. Epidermal growth factor receptor gene and protein and gefitinib sensitivity in non-small-cell lung cancer. J Natl Cancer Inst 2005; 97: 643-655

41 Chou TY, Chiu CH, Li LH et al. Mutation in the tyrosine kinase domain of epidermal growth factor receptor is a predictive and prognostic factor for gefitinib treatment in patients with non-small cell lung cancer. Clin Cancer Res 2005; 11: 3750-3757

42 Cortes-Funes H, Gomez C, Rosell R et al. Epidermal growth factor receptor activating mutations in Spanish gefitinib-treated non-small-cell lung cancer patients. Ann Oncol 2005; 16: 1081 - 1086

43 Han SW, Kim TY, Hwang PG et al. Predictive and prognostic impact of epidermal growth factor receptor mutation in non-small-cell lung cancer patients treated with gefitinib. J Clin Oncol 2005; 23: 24932501

44 Takano $T$, Ohe $Y$, Sakamoto $H$ et al. Epidermal growth factor receptor gene mutations and increased copy numbers predict gefitinib sensitivity in patients with recurrent non-small-cell lung cancer. J Clin Oncol 2005; 23: 6829-6837

45 Taron $M$, Ichinose $Y$, Rosell $R$ et al. Activating mutations in the tyrosine kinase domain of the epidermal growth factor receptor are associated with improved survival in gefitinib-treated chemorefractory lung adenocarcinomas. Clin Cancer Res 2005; 11: 5878-5885
46 Zhang XT, Li LY, Mu XL et al. The EGFR mutation and its correlation with response of gefitinib in previously treated Chinese patients with advanced non-small-cell lung cancer. Ann Oncol 2005; 16: 1334-1342

47 Mok TS, Wu YL, Thongprasert S et al. Gefitinib or carboplatin-paclitaxel in pulmonary adenocarcinoma. N Engl J Med 2009; 361: 947-957

48 Kobayashi $K$, Inoue A, Maemondo $M$ et al. First-line gefitinib versus first-line chemotherapy by carboplatin (CBDCA) plus paclitaxel (TXL) in non-small cell lung cancer (NSCLC) patients (pts) with EGFR mutations: A phase III study (002) by North East Japan Gefitinib Study Group. Proc Am Soc Clin Oncol 2009; 27: [abstr8016]

49 Tsurutani J, Mitsudomi T, Mori S et al. A phase III, first-line trial of gefitinib versus cisplatin plusdocetaxel for patients with advanced or recurrent non-small cell lungcancer (NSCLC) harboring activating mutation of the epidermal growthfactor receptor (EGFR) gene. ECCO/ESMO 2009; 7: abstr 505

50 Sequist $L V$, Bell DW, Lynch TJ et al. Molecular predictors of response to epidermal growth factor receptor antagonists in non-small-cell lung cancer. J Clin Oncol 2007; 25: 587-595

51 Inoue A, Kobayashi $K$, Usui $K$ et al. First-line gefitinib for patients with advanced non-small-cell lung cancer harboring epidermal growth factor receptor mutations without indication for chemotherapy. J Clin Oncol 2009; 27: 1394-1400

52 Cappuzzo F, Ciuleanu T, Stelmakh L et al. SATURN: A double-blind, randomized, phase III study of maintenance erlotinib versus placebo following nonprogression with first-line platinum-based chemotherapy in patients with advanced NSCLC. Proc Am Soc Clin Oncol 2009; 27: [abstr 8001]

53 Miller VA, O'Connor P, Soh C et al. A randomized, double-blind, placebocontrolled, phase IIIb trial (ATLAS) comparing bevacizumab (B) therapy with or without erlotinib (E) after completion of chemotherapy with $B$ for first-line treatment of locally advanced, recurrent, or metastatic non-small cell lung cancer (NSCLC). Proc Am Soc Clin Oncol 2009; 27: [abstr LBA8002]

54 Fidias PM, Dakhil SR, Lyss AP et al. Phase III study of immediate compared with delayed docetaxel after front-line therapy with gemcitabine plus carboplatin in advanced non-small-cell lung cancer. J Clin Oncol 2009; 27: 591 - 598

55 Weiss GJ, Rosell R, Fossella F et al. The impact of induction chemotherapy on the outcome of second-line therapy with pemetrexed or docetaxel in patients with advanced non-small-cell lung cancer. Ann Oncol 2007; $18: 453-460$

56 Brugger W, Kim J, Hansen O. Molecular markers and clinical outcome with erlotinib: results from the phase III placebo-controlled SATURN study of maintenance therapy for advanced NSCLC. J Thorac Oncol 2009; 4: S348

57 Cappuzzo F, Ciuleanu T, Steimakh L et al. Erlotinib as maintenance treatment in advanced non-small-cell lung cancer: a multicentre, randomised, placebo-controlled phase 3 study. Lancet Oncol 2010; 11: $521-529$

58 Shepherd FA, Rodrigues Pereira J, Ciuleanu Tet al. Erlotinib in previously treated non-small-cell lung cancer. N Engl J Med 2005; 353: 123-132

59 Thatcher N, Chang A, Parikh P et al. Gefitinib plus best supportive care in previously treated patients with refractory advanced non-small-cell lung cancer: results from a randomised, placebo-controlled, multicentre study (Iressa Survival Evaluation in Lung Cancer). Lancet 2005; 366: $1527-1537$

60 Bezjak A, Tu D, Seymour L et al. Symptom improvement in lung cancer patients treated with erlotinib: quality of life analysis of the National Cancer Institute of Canada Clinical Trials Group Study BR.21. J Clin Oncol 2006; 24: 3831 - 3837

61 Clark GM, Zborowski DM, Culbertson JL et al. Clinical utility of epidermal growth factor receptor expression for selecting patients with advanced non-small cell lung cancer for treatment with erlotinib. J Thorac Oncol 2006; 1: 837-846

62 Zhu CQ da Cunha Santos G, Ding K et al. Role of KRAS and EGFR as biomarkers of response to erlotinib in National Cancer Institute of Canada Clinical Trials Group Study BR.21. J Clin Oncol 2008; 26: 4268-4275

63 Cufer T, Vrdoljak E, Gaafar R et al. Phase II, open-label, randomized study (SIGN) of single-agent gefitinib (IRESSA) or docetaxel as second-line therapy in patients with advanced (stage IIIb or IV) non-small-cell lung cancer. Anticancer Drugs 2006; 17: 401-409

64 Kim ES, Hirsh V, Mok $T$ et al. Gefitinib versus docetaxel in previously treated non-small-cell lung cancer (INTEREST): a randomised phase III trial. Lancet 2008; 372: 1809-1818 
65 Niho S, Ichinose $Y$, Tamura T et al. Results of a randomized Phase III study to compare the overall survival of gefitinib (IRESSA) versus docetaxel in Japanese patients with non-small-cell lung cancer who failed one or two chemotherapy regimens. Proc Am Soc Clin Oncol 2007; 25: [abstract LBA 7509]

66 Schneider CP, Heigener D, Schott-von-Romer K et al. Epidermal growth factor receptor-related tumor markers and clinical outcomes with erlotinib in non-small cell lung cancer: an analysis of patients from german centers in the TRUST study. J Thorac Oncol 2008; 3: 1446-1453

67 Schumann C, Heigener D, Dittrich I et al. Long term benefit from Erlotinib treatment is independent of prognostic factors and therapeutic response. ECCO/ESMO 2009; 15: P 9146

68 Hughes AN, O'Brien ME, Petty WJ et al. Overcoming CYP1A1/1A2 mediated induction of metabolism by escalating erlotinib dose in current smokers. J Clin Oncol 2009; 27: 1220-1226

69 Uhm JE, Sun JM, Lee SH et al. Comparison of Erlotinib (TarcevaTM) versus Gefitinib (Iressa ${ }^{\circledR}$ ) as the Second Line Therapy for the Treatment of Advanced Non-Small Cell Lung Cancer Patients: A Randomized Phase II Trial. WCLC 2009; 13: A2.6

70 Costa DB, Schumer ST, Tenen DG et al. Differential responses to erlotinib in epidermal growth factor receptor (EGFR)-mutated lung cancers with acquired resistance to gefitinib carrying the L747S or T790M secondary mutations. J Clin Oncol 2008; 26: 1182 - 1184; author reply $1184-1186$

71 Wu JY, Shih JY, Yang CH et al. Second-line treatments after first-line gefitinib therapy in advanced nonsmall cell lung cancer. Int J Cancer 2010; 126: 247-255

72 Shukuya T, Takahashi T, Tamiya A et al. Gefitinib plus paclitaxel after failure of gefitinib in non-small cell lung cancer initially responding to gefitinib. Anticancer Res 2009; 29: 2747-2751

73 Kurata T, Tamura K, Kaneda $\mathrm{H}$ et al. Effect of re-treatment with gefitinib ("Iressa", ZD1839) after acquisition of resistance. Ann Oncol 2004; 15: $173-174$

74 Yano S, Nakataki E, Ohtsuka S et al. Retreatment of lung adenocarcinoma patients with gefitinib who had experienced favorable results from their initial treatment with this selective epidermal growth factor receptor inhibitor: a report of three cases. Oncol Res 2005; 15: 107-111

75 Yokouchi H, Yamazaki K, Kinoshita I et al. Clinical benefit of readministration of gefitinib for initial gefitinib-responders with non-small cell lung cancer. BMC Cancer 2007; 7: 51

76 Yoshimoto A, Inuzuka K, Kita T et al. Remarkable effect of gefitinib retreatment in a patient with nonsmall cell lung cancer who had a complete response to initial gefitinib. American Journal of the Medical Sciences 2007; 333: $221-225$

77 Kwak EL, Sordella R, Bell DW et al. Irreversible inhibitors of the EGF receptor may circumvent acquired resistance to gefitinib. Proc Natl Acad Sci U S A 2005; 102: 7665 - 7670

78 Li D, Ambrogio L, Shimamura T et al. BIBW2992, an irreversible EGFR/ HER2 inhibitor highly effective in preclinical lung cancer models. Oncogene 2008; 27: 4702-4711

79 Wong KK. HKI-272 in non small cell lung cancer. Clin Cancer Res 2007; 13: $s 4593$ - s4596

80 Kwak EL, Camridge DR, Clark G et al. Clinical activity observed in a phase I dose escalation trial of an oral c-met and ALK inhibitor, PF02341066. J Clin Oncol 2009; 27: abstr 3509

81 Richardson F, Richardson G, Sennello $G$ et al. Biomarker analysis from completely resected NSCLC patients enrolled in an adjuvant erlotinib clinical trial (RADIANT). Proc Am Soc Clin Oncol 2009; 25: abstr 7520 